\title{
Erratum to: Synergistic effect of aluminum and ionizing radiation upon ultrastructure, oxidative stress and apoptotic alterations in Paneth cells of rat intestine
}

\author{
N. A. Eltahawy ${ }^{1} \cdot$ S. M. Elsonbaty ${ }^{1}$ - S. Abunour ${ }^{1}$ - W. E. Zahran ${ }^{2}$
}

Published online: 25 May 2017

(C) Springer-Verlag Berlin Heidelberg 2017

Erratum to: Environ Sci Pollut Res (2017) 24:6657-6666

DOI: 10.1007/s11356-017-8392-z

The original publication of this paper contains an error.

Abstract sentence number 5 should be:

Ileum malondialdehyde, advanced oxidative protein products, protein carbonyl and tumor necrosis factor-alpha were assessed as biomarkers of lipid peroxidation, protein oxidation and inflammation respectively along with superoxide dismutase, catalase, and glutathione peroxidase activities as enzymatic antioxidants.

Abstract sentence number 8 should be:

Oxidative and inflammatory stresses assessment in the ileum of rats revealed that aluminum and ionizing radiation exposures exhibited a significant effect upon the increase in oxidative stress biomarkers along with the inflammatory marker tumor necrosis factor- $\alpha$ accompanied by a significant decreases in the antioxidant enzyme activities.

Abstract sentence number 11 should be:

The results of this study have clearly showed that aluminum and ionizing radiation exposures induced apoptosis with oxidative and inflammatory disturbance in the Paneth cells of rat intestine, which appeared to play a major role in the pathogenesis of cellular damage.

Header of Table 1 should be:

Table 1: Effect of $\gamma$-irradiation $(\gamma-\mathrm{R})$ and $\mathrm{AlCl} 3$ upon oxidative stress and inflammatory biomarkers levels in ileum of rats
The online version of the original article can be found at http://dx.doi.org/ $10.1007 / \mathrm{s} 11356-017-8392-\mathrm{z}$

\footnotetext{
W. E. Zahran

walid_zahran71@yahoo.com

1 National Center for Radiation Research and Technology, Atomic Energy Authority, Cairo, Egypt

2 Biochemistry Department, Faculty of Science, Ain Shams University, Cairo, Egypt
} 1 